\title{
A gender difference in circulating neutrophils in malnourished patients with COPD
}

This article was published in the following Dove Press journal: International Journal of Chronic Obstructive Pulmonary Disease 14 January 201 I

Number of times this article has been viewed

\author{
Sven Larsson' \\ Anita Nordenson' \\ Pernilla Glader \\ Shigemi Yoshihara ${ }^{2}$ \\ Anders Lindén' \\ Frode Slinde ${ }^{3}$ \\ 'Department of Internal \\ Medicine/Respiratory Medicine \\ and Allergology, Institute of \\ Medicine, Sahlgrenska Academy, \\ University of Gothenburg, Sweden; \\ ${ }^{2}$ Department of Pediatrics, Dokkyo \\ Medical University, Tochigi, Japan; \\ ${ }^{3}$ Department of Clinical Nutrition, \\ Institute of Medicine, Sahlgrenska \\ Academy, University of Gothenburg, \\ Sweden
}

Correspondence: Frode Slinde Department of Clinical Nutrition, Sahlgrenska Academy, University of Gothenburg, PO Box 459, SE-405 30 Göteborg, Sweden Tel +4631 7863724

Fax +46317863101

Email frode.slinde@nutrition.gu.se
Background: Circulating markers of inflammation in chronic obstructive pulmonary disease (COPD) may correlate to disease progression and extrapulmonary complications such as malnourishment. However, surprisingly little is known about gender-related differences for circulating inflammatory markers in COPD.

Purpose: To characterize differences in circulating markers of inflammation in malnourished female and male patients with COPD.

Subjects: Thirty female and 11 male patients with a clinical diagnosis of COPD and malnourishment were examined. A group of control subjects without evidence of COPD was recruited for comparison of some variables.

Methods: Blood samples were drawn, and the following parameters were studied: leukocytes and differential counts, C-reactive protein (CRP), tumor necrosis factor- $\alpha$, interleukin (IL)-6 and IL-8, myeloperoxidase (MPO), neutrophil elastase (NE), intracellular adhesion molecule-1, vascular endothelial adhesion molecule-1, and E-selectin.

Results: The mean neutrophil concentration was significantly $(P=0.019)$ higher in female $\left(4.5 \times 10^{9} / \mathrm{L}\right)$ than in male patients with COPD $\left(3.5 \times 10^{9} / \mathrm{L}\right)$ and significantly higher than in female control subjects $\left(3.1 \times 10^{9} / \mathrm{L}\right)(P<0.01, \mathrm{n}=85)$. The mean CRP values were considerably higher in female $(4.9 \mathrm{mg} / \mathrm{mL})$ than in male patients with COPD $(1.5 \mathrm{mg} / \mathrm{mL})$, but the difference was not statistically significant $(P=0.20)$. The mean concentrations of IL-6 and IL-8 tended to be higher in female than in male patients with COPD, but these differences did not reach statistical significance either $(P>0.05)$. Confounding factors (smoking, medication) could not explain the gender differences noted. The concentrations of MPO and NE displayed a strong correlation $(\mathrm{r}=0.89 ; P<0.01, \mathrm{n}=41)$ but revealed no gender differences. The latter was true for concentrations of adhesion molecules as well.

Conclusions: Our study puts forward evidence of a gender-related difference in systemic inflammation in malnourished patients with COPD in terms of circulating neutrophils being more abundant in female patients. Among these female patients, there was also a trend toward an increase in two neutrophil-mobilizing cytokines. New and better-powered studies are warranted to confirm and characterize this potentially important phenomenon in greater detail.

Keywords: chronic obstructive pulmonary disease, inflammatory markers, leukocytosis, malnutrition

\section{Introduction}

Chronic obstructive pulmonary disease (COPD) is becoming an increasing clinical problem in women. The number of females dying from COPD in the US has outnumbered that in males since the year 2000. ${ }^{1}$ In 2006, for the first time, more female (1291) 
than male (1274) patients died in Sweden from COPD, according to official death statistics. ${ }^{2}$ It seems feasible that increasing proportions of smokers among females in the generations born during the first half of the 20th century can account for the referred increasing death numbers in females. Whether females are also more susceptible to lung damage caused by cigarette smoke remains controversial; epidemiological studies are contradictory. ${ }^{3,4}$

The occurrence of systemic inflammation in COPD patients with severe disease has been reported in several studies, and COPD is now regarded as a disease with systemic complications, including an increase in circulating markers of inflammation. ${ }^{5}$ Findings of leukocytosis dominated by neutrophils and elevated levels of C-reactive protein (CRP) are frequently reported in studies on COPD patients. ${ }^{6-8}$ For example, a rapid decline in the forced expiratory volume in one second $\left(\mathrm{FEV}_{1}\right)$ in dust-exposed smokers with increased concentrations of circulating neutrophils has been reported, ${ }^{9}$ and in a group of predominantly male COPD patients, a correlation between CRP values and decline in $\mathrm{FEV}_{1}$ has been observed. ${ }^{10}$

Whether there is a difference in circulating markers of inflammation between female and male patients with COPD has been the subject of surprisingly few studies. There is one study reporting a stronger correlation between levels of circulating $\mathrm{CRP}$ and a decline in $\mathrm{FEV}_{1}$ in male than in female patients with COPD. ${ }^{11}$

In this study, we examined whether there is a genderrelated difference in circulating markers of systemic inflammation in patients with COPD and malnourishment by comparing the concentrations of circulating leukocytes, proteases, cytokines, and adhesion molecules in female and male patients with COPD and malnourishment.

\section{Methods Study subjects}

Malnourished COPD patients (body mass index $[\mathrm{BMI}]<21 \mathrm{~kg} / \mathrm{m}^{2}$ or involuntary weight loss of $>10 \%$ during the last 3 months) were included consecutively from the outpatient clinic at the Department of Respiratory Medicine at Sahlgrenska University Hospital, Gothenburg, Sweden. All patients had a clinical diagnosis of COPD and met the following inclusion criteria: a smoking history of $>10$ pack-years, age over 45 years, a spirometry test performed during the last 12 months showing an $\mathrm{FEV}_{1} / \mathrm{FVC}$ ratio less than -2 standard deviations (SD) of predicted normal and $\mathrm{FEV}_{1}<60 \%$ of predicted normal value. Every cigarette or other form of nicotine consumed and every dose of medication taken during the 24-hour period before the visit was recorded by the patients. Blood samples were taken in the morning after fasting for 12 hours. This study material was previously used for studying factors of importance to basal metabolic rate in malnourished COPD patients. ${ }^{12}$

As a control group for leukocyte, differential counts, and CRP, a study population aged 65-81 years was randomly selected from national registration. Determination of lung function through spirometry was conducted, and subjects with an $\mathrm{FEV}_{1} / \mathrm{FVC}<0.65$ were excluded (1101 subjects were invited, of whom 193 chose to take part, and 41 were excluded due to an $\mathrm{FEV}_{1} / \mathrm{FVC}<0.65$ indicating COPD). Smoking habits were recorded.

\section{Blood sampling and analysis of systemic inflammatory markers}

A volume of $42 \mathrm{~mL}$ of blood was collected from each study subject. Serum was prepared for analysis of soluble inflammatory markers, and cell differential counts were performed as described below.

The concentration of CRP was measured utilizing a high sensitivity latex reagent kit (hs-CRP; Roche Diagnostics, Mannheim, Germany) using a modular polypeptide analyzer (Roche, Tokyo, Japan) at the accredited laboratory at the Department of Clinical Chemistry, Sahlgrenska University Hospital. The intra-assay coefficient of variation of the CRP method was $2 \%$ at $1 \mathrm{mg} / \mathrm{L}$ and $1 \%$ at $20 \mathrm{mg} / \mathrm{L}$. The concentration of leukocytes was obtained from an automated blood differential count analysis at the same laboratory. Commercially available enzyme-linked immunosorbent serologic assay (ELISA) kits were utilized to analyze the serum concentrations of tumor necrosis factor (TNF)- $\alpha$ (Catalog No. HSTA00D; R\&D Systems, Abingdon, England) and interleukin (IL)-6 (Catalog No. HS600B; R\&D Systems) and IL-8 (Catalog No. D800C; R\&D Systems). The concentration of myeloperoxidase (MPO) in serum was also measured using ELISA (HK327; Hycult Biotechnologies, Uden, the Netherlands), in this case after diluting the serum 1:10. The concentration of neutrophil elastase $(\mathrm{NE})(\mu \mathrm{g} / \mathrm{L})$, was assessed using the latex bead concentration method as previously described. ${ }^{13,14}$ The adhesion molecules intracellular adhesion molecule (ICAM)-1, vascular endothelial adhesion molecule (VCAM)-1, and E-selectin were measured using ELISA (Diaclone, Besancon, France) as well.

\section{Ethics}

The Committee for Medical Research Ethics at the University of Gothenburg approved the study (diary number S349-03). 
The patients were informed of the nature and purpose of the study. Oral as well as written informed consent was obtained.

\section{Statistics}

Parametric, descriptive statistics were used to describe the study population. Two-sided $t$-tests were used to compare groups. Clinical characteristics were used as independent predictors of neutrophil count in univariate regression analysis. Variables that were associated with neutrophil count with a $P<0.15$ were, in addition to gender, included in a multivariate linear forward regression analysis. All statistical analysis was performed using PASW Statistics 18.0.0 (SPSS Inc., Chicago, IL, USA).

\section{Results}

The clinical characteristics of the patients (11 males and 30 females) are presented in Table 1. BMI and the amount of cigarettes smoked (pack-years) were significantly lower in female compared with male patients. No other statistically significant gender-related differences were observed with reference to the referred clinical characteristics. Slightly fewer female than male patients used $\beta_{2}$-adrenoceptor agonists and inhaled steroids. Three patients, all female, used oral steroids. The clinical characteristics of the control group are presented in Table 2.

The serum concentrations of inflammatory cells and inflammatory markers in male and female patients are presented in Table 3. The mean concentration of circulating neutrophils was significantly higher in female than in male patients $(P=0.019)$. The mean corresponding CRP concentration was also considerably higher in female than in male patients, but this difference did not reach

Table I Descriptives of COPD cases (mean [SD])

\begin{tabular}{llll}
\hline & $\begin{array}{l}\text { Males } \\
(\mathbf{n}=\mathrm{II})\end{array}$ & $\begin{array}{l}\text { Females } \\
(\mathbf{n}=\mathbf{3 0})\end{array}$ & $\boldsymbol{P}$ \\
\hline Age (years) & $65(9)$ & $63(9)$ & 0.47 \\
Body mass index $\left(\mathrm{kg} / \mathrm{m}^{2}\right)$ & $19.8(\mathrm{I} . \mathrm{I})$ & $18.5(2 . \mathrm{I})$ & 0.045 \\
$\mathrm{FEV}_{1}$ (percentage predicted) & $36(\mathrm{I5})$ & $40(\mathrm{I5})$ & 0.48 \\
$\mathrm{PO}_{2}$ & $9.8(0.9)$ & $9.2(\mathrm{I} .5)$ & 0.27 \\
$\mathrm{PCO}_{2}$ & $5.6(0.6)$ & $5.7(0.6)$ & $0.6 \mathrm{I}$ \\
Pack-years & $47(\mathrm{I3})$ & $34(10)$ & $<0.0$ I \\
Percentage of current smokers & 36 & 37 & \\
Percentage of $\beta 2$-adrenoceptor & 82 & 60 & \\
agonists users & & & \\
Percentage of inhalation steroid users & 91 & 77 & \\
Percentage of anticholinergic drug users & 73 & 80 & \\
\hline
\end{tabular}

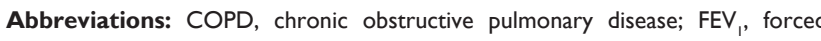
expiratory volume in one second; $\mathrm{PO}_{2}$, partial pressure of oxygen in arterial blood; $\mathrm{PCO}_{2}$, partial pressure of carbon dioxide in arterial blood; SD, standard deviation.
Table 2 Descriptives of the control group (mean [SD]) $(n=\mid 5 I)$

\begin{tabular}{llll}
\hline & $\begin{array}{l}\text { Males } \\
(\mathbf{n}=66)\end{array}$ & $\begin{array}{l}\text { Females } \\
(\mathbf{n}=\mathbf{8 5})\end{array}$ & $\mathbf{P}$ \\
\hline Age (years) & $72(5)$ & $72(5)$ & 0.43 \\
Body mass index $\left(\mathrm{kg} / \mathrm{m}^{2}\right)$ & $26.0(3.0)$ & $25.5(3.8)$ & 0.37 \\
FEV (percentage predicted) & $102(15)$ & $107(17)$ & 0.053 \\
Percentage of current smokers & 5 & 12 & - \\
\hline
\end{tabular}

Abbreviations: $\mathrm{FEV}_{1}$, forced expiratory volume in one second; SD, standard deviation.

statistical significance. No other gender-related difference was observed with reference to clinical characteristics.

The concentration of circulating neutrophils in female COPD patients was clearly higher than in female control subjects (4.5 compared with $3.1 \times 10^{9} / \mathrm{L}, P<0.01, \mathrm{n}=85$ ). No corresponding difference was observed for male patients and control subjects (3.5 compared with $3.1 \times 10^{9} / \mathrm{L}$, $P=0.29, \mathrm{n}=66$ ). In addition, the concentration of circulating CRP was significantly higher in female patients compared with female control subjects (4.9 compared with $2.3 \mathrm{mg} / \mathrm{mL}$, $P=0.023, \mathrm{n}=85$ ). With regard to CRP in males, the concentration tended to be lower in patients with COPD than in healthy control subjects, but this difference did not reach statistical significance (1.5 compared with $3.9 \mathrm{mg} / \mathrm{mL}$, $P=0.43, \mathrm{n}=66)$.

The variables that were associated with neutrophil concentrations with a $P<0.15$ in univariate regression

Table 3 Inflammatory cells and inflammatory markers in serum of COPD patients (mean [SD])

\begin{tabular}{|c|c|c|c|}
\hline & $\begin{array}{l}\text { Males } \\
(n=I I)\end{array}$ & $\begin{array}{l}\text { Females } \\
(n=30)\end{array}$ & $P^{a}$ \\
\hline Monocytes $\left(\times 10^{9} / \mathrm{L}\right)$ & $0.65(0.15)$ & $\begin{array}{l}0.63(0.16) \\
(\mathrm{n}=29)\end{array}$ & 0.75 \\
\hline Lymphocytes $\left(\times 10^{9} / \mathrm{L}\right)$ & $2.3(1.1)$ & $\begin{array}{l}2.3(0.8) \\
(n=29)\end{array}$ & 0.89 \\
\hline Neutrophils $\left(\times 10^{9} / \mathrm{L}\right)$ & $3.5(0.7)$ & $\begin{array}{l}4.5(1.2) \\
(n=29)\end{array}$ & 0.019 \\
\hline CRP (mg/mL) & $1.5(1.5)$ & $4.9(8.6)$ & 0.20 \\
\hline IL-6 (pg/mL) & $3.6(1.8)$ & $3.9(2.5)$ & 0.75 \\
\hline TNF- $\alpha(p g / m L)$ & $2.5(2.5)$ & $1.6(1.4)$ & 0.17 \\
\hline IL-8 (pg/mL) & I5.I (4.4) & $17.7(7.7)$ & 0.30 \\
\hline MPO (ng/mL) & $165(114)$ & $153(116)$ & 0.75 \\
\hline NE (ng/mL) & $243(201)$ & $246(235)$ & 0.98 \\
\hline ICAM-I (ng/mL) & $683(138)$ & 734 (I72) & 0.38 \\
\hline VCAM-I (ng/mL) & $940(202)$ & $993(264)$ & 0.55 \\
\hline E-selectin $(\mathrm{ng} / \mathrm{mL})$ & $75(39)$ & $\begin{array}{l}66(28) \\
(n=29)\end{array}$ & 0.39 \\
\hline
\end{tabular}

Note: ${ }^{\text {at }}$-test.

Abbreviations: COPD, chronic obstructive pulmonary disease; CRP C-reactive protein; ICAM, intracellular adhesion molecule; IL, interleukin; MPO, myeloperoxidase; NE, neutrophil elastase; SD, standard deviation; TNF, tumor necrosis factor; VCAM, vascular endothelial adhesion molecule. 
analysis were NE, MPO, TNF- $\alpha$, pack-years, and BMI. The result from the multivariate linear forward regression analysis is presented in Table 4. Only gender and MPO significantly explained the variation in neutrophil concentration. NE, TNF- $\alpha$, pack-years, and BMI did not increase the degree of explanation of the variation in neutrophil concentration. The neutrophil concentrations of the different subgroups are presented in Table 5. Notably, there were no significant differences between smokers and exsmokers, nicotine users and nonusers, or patients using or not using $\beta_{2}$-adrenoceptor agonists or inhaled steroids. The concentration of circulating neutrophils in users of anticholinergic drugs was considerably (but not statistically significantly) higher than in nonusers. Of the exsmoking nicotine users, two patients used nicotine patches, two patients used nicotine chewing gum, and five patients used snuff. There was no difference in neutrophil concentration between snuff users and nonsnuffers (a mean concentration of $4.2 \times 10^{9} / \mathrm{L}$ in both groups).

A scatter plot for MPO and NE concentrations is presented in Figure 1, and the correlation for these two outcomes was strong and statistically significant $(\mathrm{r}=0.89$; $P<0.01, \mathrm{n}=41$ ).

We found no statistically significant differences for the female and male COPD patients with respect to concentrations of adhesion molecules (Table 3 ).

\section{Discussion}

In this observational study, we found significantly higher concentrations of circulating neutrophils in female than in male COPD patients with malnourishment. We could not identify any confounding factor explaining this difference, taking smoking, use of nicotine, and medication into account. The only variable identified other than gender related to neutrophil concentrations in the forward regression model was the concentration of MPO in serum. This is not surprising, because MPO might be produced by neutrophils. ${ }^{15}$ Users of inhaled steroids had marginally higher neutrophil concentrations than nonsteroid users, in line with previous studies. ${ }^{16}$ Notably, fewer female than male patients used inhaled steroids.

Table 4 Results from the multivariate linear forward regression, $r^{2}=0.25$

\begin{tabular}{llll}
\hline Variable & B & Standard error & P-value \\
\hline Intercept & 2.8 & 0.4 & $<0.001$ \\
Gender & I.I & 0.4 & $0.006 \mathrm{I}$ \\
MPO & 0.0045 & 0.0019 & 0.021 \\
\hline
\end{tabular}

Abbreviation: MPO, myeloperoxidase.
Table 5 Number of neutrophils in different subgroups of the population (mean $[S D]$ )

\begin{tabular}{lll}
\hline & $\begin{array}{l}\text { Neutrophils } \\
\left(\times 10^{9} / \mathbf{L}\right)\end{array}$ & $P^{a}$ \\
\hline Males $(n=I I)$ & $3.5(0.7)$ & 0.019 \\
Females $(n=29)$ & $4.5(I .2)$ & \\
Smokers $(n=15)$ & $4.3(I . I)$ & 0.65 \\
Exsmokers $(n=25)$ & $4.2(I .3)$ & \\
Nicotinists $(n=24)$ & $4.2(I .2)$ & 0.99 \\
Non-nicotinists $(n=16)$ & $4.2(I .2)$ & \\
Users of $\beta_{2}$-adrenoceptor agonists $(n=27)$ & $4.1(I .3)$ & 0.27 \\
Nonusers of $\beta_{2}$-adrenoceptor agonists $(n=13)$ & $4.5(0.8)$ & \\
Users of inhalation steroids $(n=32)$ & $4.3(I .2)$ & 0.70 \\
Nonusers of inhalation steroids $(n=8)$ & $4.1(I .1)$ & \\
Users of anticholinergic drug $(n=32)$ & $4.4(I .2)$ & 0.060 \\
Nonusers of anticholinergic drug $(n=8)$ & $3.5(I .0)$ & \\
\hline
\end{tabular}

Note: ${ }^{a} t$-test.

Abbreviation: SD, standard deviation.

Thus, the difference between users and nonusers of inhaled steroids cannot explain the gender difference in neutrophil concentrations in our study. Just like the use of inhaled steroids, the use of oral steroids will increase neutrophil concentrations. ${ }^{17}$ The key results of our current study did not, however, change in any way if the three patients taking oral steroids were excluded. There were also other small gender differences concerning pharmacological therapy. Fewer females than males used $\beta_{2}$-adrenoceptor agonists. On the other hand, more female patients used anticholinergic drugs compared with male patients. The reason for these differences and their possible effects on neutrophil concentrations remains unclear. The finding of a considerably, but not significantly,

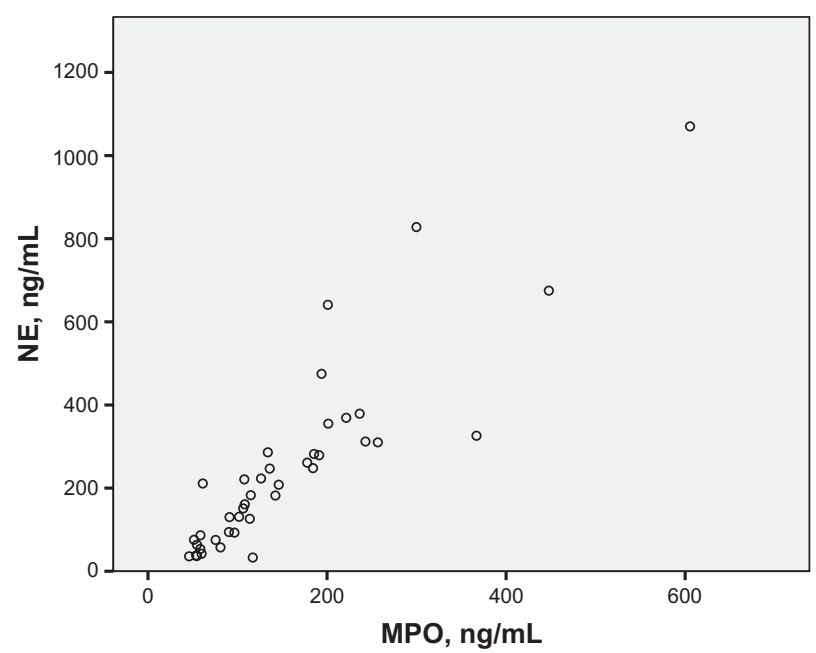

Figure I Relation between MPO and NE in serum $(r=0.89 ; P<0.01, n=4 I)$ in malnourished COPD patients.

Abbreviaions: COPD, chronic obstructive pulmonary disease; MPO, myeloperoxidase; NE, neutrophil elastase. 
higher concentration of circulation neutrophils in users of anticholinergic drugs compared with nonusers is somewhat surprising. Anticholinergic drugs are supposed to ameliorate neutrophilic inflammation. ${ }^{18}$

Our finding of significantly elevated neutrophil concentrations in female COPD patients must be interpreted with care, as this outcome was not the primary study variable of this investigation. However, in another, ongoing study of COPD patients (including patients with normal weight as well as malnourished patients) we found the same pattern (unpublished observations). The preliminary results of the referred, ongoing study thus support our current study.

Notably, we observed no clear difference in neutrophil concentrations for female and male control subjects. In line with this, clinically applied normal values for neutrophils do not differ for female and male subjects either. The comparison with the control group also indicates that the gender difference noted in the COPD patients is caused by increased neutrophil concentration in females rather then decreased concentrations in men.

We determined the concentrations of the neutrophilmobilizing cytokines IL-6 and IL-8 to look for molecular correlates that could explain the increased neutrophil concentration in the female COPD patients included in our current study. Whereas the concentrations of both these cytokines tended to be higher among female patients, the difference compared with male patients did not reach statistical significance. Even though not conclusive, these findings are compatible with the archetype chemokine IL- 8 and the neutrophil-activating cytokine IL-6, contributing to a gender difference in the concentration of circulating neutrophils, and it can be speculated that a better-powered study may prove this to be the case in the future.

Estrogens reduce the expression of adhesion molecules, leading to less extravasation of inflammatory cells. ${ }^{19}$ To study the hypothesis that the higher serum concentrations of neutrophils in female COPD patients were caused by low expression of adhesion molecules, which would reduce the capacity for extravascular migration, we studied ICAM-1, VCAM-1, and E-selectin. Hypothetically, a low expression of these molecules would lead to a larger fraction of neutrophils remaining in the circulation. However, we obtained no evidence supporting this hypothesis. On the contrary, the mean concentration of ICAM-1 and VCAM-1, respectively, was higher in female than in male patients, although not significantly so, suggesting more expression of these molecules at the cellular level, if anything. Furthermore, there was no correlation between the concentrations of inflammatory cells and circulating adhesion molecules.

The concentrations of circulating MPO and NE were determined to assess whether the increased concentrations of circulating neutrophils in female COPD patients were associated with an increased release of neutrophil-derived proinflammatory compounds. No evidence for this was found, however. There were no clear differences in MPO and NE concentrations between female and male COPD patients. The concentrations of MPO and NE did, however, display a very strong correlation to one another. That finding is compatible with a shared source at the cellular level. However, this source may not be the circulating neutrophil, as no correlation was found between MPO or NE, on the one hand, and the neutrophil concentration, on the other hand. We speculate that neutrophils residing in the lung tissue could be one possible source.

The concentrations of circulating CRP were considerably higher in female than in male patients with COPD and significantly higher in female patients than in female control subjects. Although the gender difference in subjects with COPD was not statistically significant, this is of concern. This is because, in elderly subjects, slightly elevated concentrations of CRP are reported as predictors of frailty and osteoporosis..$^{20}$ Elevated CRP concentrations are also correlated to an increased risk of cardiovascular mortality. ${ }^{21}$ In the general elderly population in Sweden, there is no difference in CRP concentrations for female and male subjects. ${ }^{22}$

\section{Conclusion}

Data show that the concentration of circulating neutrophils is higher in female than in male COPD patients with malnourishment. This difference is not explained by differences in smoking habits, use of nicotine, or inhalation steroids. The results are compatible with, but do not prove, the idea that concentrations of circulating IL-6 and IL-8 relate to the observed difference in neutrophils, and this clearly deserves to be evaluated in better-powered studies in the future. This type of gender difference in circulating markers of inflammation may prove important to understanding the link between disease progression and systemic complications in COPD and may lead to new therapeutic targets as well.

\section{Acknowledgments}

We thank Bengt Andersson MD, PhD and Mirjana Hahn-Zoric MD, PhD for their skillful assistance with laboratory analyses. 


\section{Disclosure}

The authors are grateful to the Swedish Research Council (K2008-57x-09048-19-3), Swedish Heart-Lung Foundation, Swedish Heart and Lung Association, and Swedish Nutrition Foundation for financial support. The work was also supported by federal grants according to the ALF/LUA agreement. No funding was obtained from the tobacco industry or the food industry.

\section{References}

1. Mannino DM, Homa DM, Akinbami LJ, Ford ES, Redd SC. Chronic obstructive pulmonary disease surveillance-United States, 1971-2000. MMWR Surveill Summ. 2002;51:1-16.

2. The National Board of Health and Welfare (Socialstyrelsen). Hälso- och sjukvårdsrapport 2009. Socialstyrelsen, Stockholm, Sweden, 2009.

3. Cote CG, Chapman KR. Diagnosis and treatment considerations for women with COPD. Int J CLin Pract. 2009;63:486-493.

4. DeTorres JP, Campro A, CasanovaC, Aguirre-Jaime A, Zuluenta J. Gender and chronic obstructive pulmonary disease in high risk smokers. Respiration. 2006;73:306-310.

5. Wouters E. COPD: from obstructive lung disease to chronic systemic inflammatory syndrome? Pneumologie. 2009;63(Suppl 2):S107-S112.

6. Broekhuizen R, Wouters EFM, Creutzberg EC, Schols AM. Raised CRP levels mark metabolic and functional impairment in advanced COPD. Thorax. 2006;61:17-22.

7. Pinto-Plata VM, Mullerova H, Toso JF, et al. C-reactive protein in patients with COPD, control smokers and non-smokers. Thorax. 2006;61:23-28.

8. Gan WQ, Man SF. Association between chronic obstructive pulmonary disease and systemic inflammation: a systematic review and a metaanalysis. Thorax. 2004;59:574-580.

9. Chan-Yeung M, Abboud R, Buncio AD, Vedal S. Peripheral leucocyte count and decline in lung function. Thorax. 1988;43:462-466.

10. Higashimota Y, Iwata T, Okada M, Satoh H, Fukuda K, Tohda Y. Serum biomarkers as predictors of lung function decline in chronic obstructive pulmonary disease. Resp Med. 2009;103:1231-1238.
11. Olafsdottir IS, Gislason T, Thjodleifsson B, et al. Gender differences in the association between C-reactive protein, lung function impairment, and COPD. Int J Chron Obstruct Pulmon Dis. 2007;2:635-642.

12. Nordenson A, Grönberg AM, Hulthén L, Larsson S, Slinde F. A validated disease specific prediction equation for resting metabolic rate in underweight patients with COPD. Int J COPD. 2010;5:271-276.

13. Hafner G, Erbes H, Drosdat H, Lotz J, Ehrental W, Wurzburg U. Evaluation of a new assay for the determination of PMN elastase- $\alpha-1$ proteinase inhibitor complexes in EDTA and citrated plasma. Clin Laboratory. 1997;43:3-9.

14. Yoshihara S, Yamada Y, Abe T, Lindén A, Arisaka O. Association of epithelial damage and signs of neutrophil mobilization in the airways during acute exacerbations of pediatric asthma. Clin Exp Immunol. 2006; 144:212-216.

15. Furth V. Development and distribution of mononuclear phagocytes. In: Gallin JIGI, Snyderman R, editors. Inflammation. Basic Principles and Clinical Correlates. 2nd ed. New York: Raven Press;1992:325-51.

16. Blaiss MS, Herrod HG, Crawford LV, Lieberman PL. Beclomethasone dipropionate aerosol: hematologic and immunologic effects. Ann Allergy. 1982;48:210-214.

17. Bishop CR, Athens JW, Boggs DR, Warner HR, Cartwright GE, Wintrobe MM. Leukokinetic studies: a non-steady-state kinetic evaluation of the mechanism of cortisone-induced granulocytosis. $J$ Clin Invest. 1968;47:249-260.

18. Wollin L, Pieper MP. Tiotropium bromide exerts anti-inflammatory activity in a cigarette smoke mouse model of COPD. Pulm Pharmacol Ther. 2010;23:345-354

19. Nilsson BO. Modulation of the inflammatory response by estrogens with focus on the endothelium and its interactions with leukocytosis. Inflamm Res. 2007;56:269-273.

20. Barzilay J, Blaum C, Moore T. Insulin resistance and inflammation as precursors of frailty: the cardiovascular health study. Arch Intern Med. 2007;167:635-641.

21. Emerging Risk Factors Collaboration, Kaptoge S, di Angelantonio E, et al. C-reactive protein concentration and risk of coronary heart disease, stroke, and mortality: an individual participant meta-analysis. Lancet. 2010;9:132-140.

22. Carlsson L, Lind L, Larsson A. Reference values for 27 clinical chemistry tests in 70-year-old males and females. Gerontology. 2009;56: $259-265$.
International Journal of COPD

\section{Publish your work in this journal}

The International Journal of COPD is an international, peer-reviewed journal of therapeutics and pharmacology focusing on concise rapid reporting of clinical studies and reviews in COPD. Special focus is given to the pathophysiological processes underlying the disease, intervention programs, patient focused education, and self management

\section{Dovepress}

protocols. This journal is indexed on PubMed Central, MedLine and CAS. The manuscript management system is completely online and includes a very quick and fair peer-review system, which is all easy to use. Visit http://www.dovepress.com/testimonials.php to read real quotes from published authors. 